\title{
Editorial
}

\section{Lipoprotein alterations in diabetes mellitus}

\author{
G. M. Kostner ${ }^{1}$ and I. Karádi ${ }^{2}$ \\ ${ }^{1}$ Institute of Medical Biochemistry, University of Graz, Austria, and \\ ${ }^{2}$ Third Department of Medicine, Semmelweis Medical School, Budapest, Hungary
}

Long term diabetes mellitus is known to be accompanied by atherosclerotic vascular diseases $[1,2]$. The lipoprotein alterations found in diabetic patients appear to contribute to, and are partly responsible for the development of early atherosclerosis [3-6]. Many attempts have been made to clarify the exact mechanisms of disturbed lipoprotein metabolism and a lot of data based on several experimental and clinical observations are available [21]. More recent studies, which have utilised different methods investigating the apoprotein composition and the receptor-mediated uptake of lipoproteins by different tissue cells clearly indicate that there exists no specific diabetic lipoprotein alteration common to all diabetic patients. Without doubt, there is a proven relationship between the degree of diabetic control and plasma lipid levels, but epidemiological studies have also revealed an increased risk for atherogenesis even in persons with minimal alterations in glucose tolerance [7-10]. Thus, poor metabolic control per se does not give a clear explanation of the lipoprotein abnormalities found in diabetic patients. The purpose of this editorial is to provide a comprehensive overview of diabetic dyslipoproteinaemia and discuss its metabolic aspects in detail. Diabetes mellitus exists in two forms, one characterised by insulin deficiency, Type 1 (insulin-dependent) diabetes mellitus, and the other by tissue resistance to insulin, Type 2 (non-insulin-dependent) diabetes mellitus. Considering that these two types of diabetes mellitus differ from one another in several aspects, we have presented, in the following, problems of lipoprotein metabolism for both diseases separately.

\section{The role of insulin in synthesis and degradation of lipoproteins}

Among others, one of the important functions of insulin is to coordinate the conversion of dietary carbohydrates to internal stored energy as fat [11]. Under normal circumstances insulin influences the lipoprotein metabolism through different metabolic routes.
After the absorption of fat from the gut most of the triglycerides entering the plasma from intestinal lymph are found in chylomicrons; but after induction of insulin deficiency by streptozotocin in animals, there is an increase of intestinal very low density lipoprotein (VLDL)-triglyceride production as well [12-15]. These studies may suggest that the intestine participates in the production of VLDL under the control of insulin.

The VLDL and chylomicrons, as carriers of endogenous and exogenous triglycerides, are substrates for the lipoprotein lipase system. With a lack of insulin the activity of lipoprotein lipases decreases [16-18]. Since the work of Bagdade et al. [19] it seems clear that this is one of the major causes of hyperlipaemia in poorly controlled diabetic patients. The other enzyme, which is involved in the metabolism of triglyceriderich particles is the hepatic triglyceride lipase; its insulin sensitivity also determines the lipoprotein abnormalities found in diabetes mellitus $[20,21]$. There is a possibility that a decreased activity of hepatic lipase may interfere mainly with remnant clearance [22].

The second step in the metabolism of triglyceriderich lipoproteins is the receptor-mediated removal of remnants from the plasma [23]. Under the action of lipoprotein lipases, the VLDL and chylomicron particles lose their triglyceride content and turn into a cholesterylester-rich form. These so-called remnants contain apoprotein $\mathrm{E}$, which is mainly responsible for their receptor-mediated uptake by the liver. The role of hepatic apoprotein- $E$ receptors is well known, but how insulin influences their activity remains to be clarified.

There are several controversial aspects relating to whether or not insulin contributes to hepatic VLDLtriglyceride secretion. The exact mechanism by which insulin stimulates lipoprotein synthesis in the liver is not well understood. The most plausible explanation is based on the premise that elevated plasma non-esterified fatty acid (NEFA) concentration, even at normal insulin levels results in an increased hepatic VLDLtriglyceride secretion concomitant with elevated plas- 
ma triglyceride concentrations $[24,25]$. High NEFA levels can be observed in patients with poorly controlled Type 2 diabetes, who have lost the ability to counterbalance the decreased peripheral glucose uptake by hyperinsulinism and have nearly normal insulin concentration. If so, then they are also resistant to the ability of normal insulin levels to regulate plasma NEFA metabolism and therefore have high plasma NEFA levels [25]. There is evidence that an increased level of insulin may both enhance, and sometimes inhibit the triglyceride-rich lipoprotein secretion of the liver [26].

The cholesterol content of plasma originates from two main sources; the first, from cholesterol absorbed from the diet; and second, from "de novo" cholesterol synthesis of different tissues [23]. The esterified cholesterol that appears in cholesterol-rich lipoproteins (LDL, IDL) arises from free cholesterol uptake by HDL from peripheral cells followed by esterification through lecithin: cholesterol acyltransferase and transfer to lipoproteins of lower density by specific proteins. This peripheral cholesterol removal may be impaired by the lack of insulin [27, 28]. On the basis of studies with human skin fibroblasts it has been suggested that the LDL-receptor activity, including the internalisation process may be enhanced by insulin [29]. The maintenance of normoglycaemia, as an evidence of successful insulin action provides protection against the modification of lipoproteins (mainly LDL) by nonenzymatic glycosylation [30-35]. Finally, insulin helps to balance the apoprotein composition of lipoproteins involved in enzymatic activation (e.g. apoprotein A-I/ A-II and apoprotein C-II/C-III ratio) and receptormediated uptake of lipoproteins (apoprotein B and $\mathrm{E}$ contents) [36-43].

\section{Alteration of lipoproteins in Type 1 (insulin-dependent) diabetes mellitus}

The pathophysiology of Type 1 diabetes mellitus is explained by the diminished insulin secretion by the Langerhans islet cells of the pancreas. Insulin deficiency in a poor metabolic state results in a marked hypertriglyceridaemia mainly due to an ineffective activation of the lipoprotein lipase system [44]. The elimination of VLDL and chlylomicrons decreases and, especially in diabetic ketoacidosis, a marked elevation of triglyceride-rich lipoproteins can be observed. Excess of NEFA's in a poorly controlled metabolic state contribute to the elevation of hepatic triglyceride secretion [45]. Later, in a severe ketoacidotic state the enhanced VLDL secretion falls off as a result of decreased hepatic protein synthesis [46]. Elevated VLDL-, LDLand HDL-triglycerides exist in Type 1 diabetes mellitus during insulin deficiency. The high proportions of triglycerides in VLDL and LDL are due to an accumulation of triglycerides in these particles, but the cause of the elevated HDL-triglyceride is a result of an exchange of cholesteryl esters for triglycerides between HDL and VLDL and LDL, mediated by the cholesteryl ester transfer protein [28].

There is little doubt that insulin deficiency (concerning the effect of insulin on receptorial uptake and non-enzymatic glycosylation of LDL) causes an increase in the LDL level, too. An increased cholesterol to apoprotein $\mathrm{B}$ ratio has been demonstrated in LDL of Type 1 patients; the LDL particles from Type 1 individuals are taken up at a lower rate than LDL molecules of normal subjects $[38,47]$. However, the effect of improved metabolic control on plasma cholesterol and LDL level in Type 1 patients is a matter of debate. Pietri et al. [48] showed that it depends on the interpretation of "good metabolic control". In their study using a continuous subcutaneous insulin infusion system (CSII) they have shown the benefit of exact glucose control in so-called "well controlled" Type 1 patients on conventional insulin therapy. A further decrease of plasma cholesterol and LDL-cholesterol levels could be observed under perfect insulin treatment.

Screening of lipoprotein levels in several larger Type 1 diabetes group studies has shown that the total cholesterol, total triglycerides and LDL-cholesterol levels in well controlled Type 1 patients do not differ from those of control, non-diabetic individuals [49, 50]. However, distinct differences between Type 1 patients with and without coronary heart disease (CHD) have been observed. In the study of Laakso et al. [51] the Type 1 patients with CHD had significantly higher total triglycerides, LDL-cholesterol, VLDL-triglycerides and lower HDL-cholesterol levels than Type 1 diabetic patients without CHD. The lipoprotein profile of Type 1 patients with CHD was similar to that of non-diabetic patients with CHD. It is possible that these small but distinct alterations may have a predictive value with respect to the risk of CHD in Type 1 patients.

Data from some reports serve as evidences for normal, or sometimes higher than normal HDL-cholesterol levels in insulin treated diabetic patients in a good metabolic state [52, 53]. The lipoprotein lipase activity is usually also elevated in these patients, possibly caused by injection of external insulin to peripheral tissues; this extra-hepatic action of insulin promotes the catabolism of triglyceride-rich lipoproteins and subsequently may increase the HDL level $[53,54]$. The rise of HDL-cholesterol is accounted for mostly by an increase in $\mathrm{HDL}_{2}$-cholesterol subfraction $[55,56]$.

The high apoprotein A-I plasma level found in poorly controlled Type 1 patients is a direct sign of lipoprotein lipase deficiency [57]. During hydrolysis of chylomicrons and VLDL the surface material containing apoprotein A-I is transfered to $\mathrm{HDL}[57,58]$. The accumulation of triglyceride-rich particles results in an increment of apoprotein A-I and apoprotein B content of these lipoproteins as a consequence of delayed 
removal of lipoproteins with a density of 1.019. Data indicate that there may be alterations in the composition of HDL in Type 1 diabetes mellitus. Triglyceriderich $\mathrm{HDL}_{3}$ particles smaller in size have been shown [59]. Decreased apoprotein A-I and apoprotein A-II content of HDL have been reported as well [60].

In general, the changes in lipoprotein composition in Type 1 diabetes mellitus depend on the degree of glycaemic control. In a poor metabolic state the VLDL, LDL and HDL become rich in triglycerides and an overproduction of VLDL and LDL and a decreased removal of VLDL remnants can be found. Lower than normal cholesterol to apoprotein $\mathrm{B}$ ratio can be measured in LDL and the receptorial uptake of altered LDL particles is diminished. In well controlled Type 1 patients the single alteration may only be the slightly elevated HDL-cholesterol level due to higher peripheral insulin concentration causing enhanced lipoprotein lipase activity.

From a clinical point of view, the lipoprotein composition in insulin-treated diabetic patients can change in an atherogenic direction in the presence of obesity, poor metabolic control or diabetic nephropathy [53]. A 10-fold increased cardiovascular mortality can be found in Type 1 diabetic patients with diabetic nephropathy. Jensen et al. [61] studied Type 1 diabetic patients with increased urinary albumin secretion. In patients with incipient nephropathy the serum cholesterol, triglycerides, LDL, VLDL-cholesterol and fibrinogen levels were increased by $11-14 \%$ compared to Type 1 diabetic patients without proteinuria. It thus seems, that nephropathy in Type 1 diabetes mellitus is closely associated with an increase of apoprotein B containing lipoproteins, notably of VLDL, IDL and LDL, as well as of fibrinogen, which may well explain by itself the increased risk for atherosclerosis.

\section{Type 2 (non-insulin-dependent) diabetes mellitus and dyslipoproteinaemia}

In contrast to Type 1 diabetes mellitus, in diabetes treated with diet or oral antidiabetic agents (OAD), the insulin levels in portal plasma are higher than in peripheral plasma and the metabolic disturbances are focussed on the liver [25]. Hepatic hyperinsulinaemia leads to an overproduction of VLDL and triglycerides, but the relative peripheral insulin deficiency results in a depressed catabolism of triglyceride-rich lipoproteins [53]. Due to methodological problems the interpretations of the disturbances in the lipoprotein lipase system are not entirely consistent in Type 2 diabetes mellitus. Decreased acute and late lipoprotein lipase activity have been found, and a diminution in adipose tissue lipoprotein lipase in Type 2 diabetes mellitus has been described $[44,62,63]$. Despite these observations the exact pathophysiological significance of lipoprotein alterations has not been established. However, the decreased activity of lipoprotein lipase is consistent with the decrease in the clearance of VLDL, and improved metabolic control followed by elimination of hyperglycaemia results in an increased lipoprotein lipase activity.

High plasma totals and VLDL triglycerides can be found in a large number of these patients (approximately $20-60 \%)[16,18,52]$. The marked hypertriglyceridaemia existing in some Type 2 patients suggests the role of other genetic defects in lipoprotein metabolism $[64,65]$. In these patients, at a nearly normoglycaemic state pathological triglyceride levels have been observed.

There are indications that, disregarding the high triglyceride content, other alterations in particle composition of VLDL exist as well. Increases in the proportions of apoprotein $\mathrm{C}$ and $\mathrm{E}$ compared to other components of VLDL in Type 2 diabetes mellitus have been demonstrated $[36,66]$. This may result in an inhibition or retardation of liver uptake, but the observation in in vitro studies have confirmed an inverse correlation between the uptake of VLDL in liver and apoprotein $\mathrm{C}$ content [67]. Other investigators could not show any changes in the apoprotein C-II/C-III ratio. These two apoproteins have the greatest influence on lipoprotein lipase activity. The apoprotein $\mathrm{C}$-II acts as an activator; and C-III is a well known inhibitor of this enzyme. Another interesting observation indicates that VLDL particles isolated from Type 2 diabetic patients show a significantly greater accumulation in mouse peritoneal macrophages than did VLDL molecules from non-diabetic control patients [25]. Macrophages containing high amounts of fat may be transformed into foam cells, which can be found in great numbers in the atherosclerotic fibrous plaque. The details and conditions of this transformation are not known.

Generally, nearly normal LDL levels have been shown by several authors in Type 2 diabetes mellitus. The concentration of LDL is influenced by different metabolic effects and the final level depends on the rate of synthesis as well as catabolism. In severe hyperglycaemia a decreased fractional catabolic rate of apoprotein B in LDL has been demonstrated in Type 2 diabetic patients. Despite decreased clearance the LDL levels in such patients appeared to be normal. The explanation of this phenomenon can be found in VLDL metabolism. The composition of VLDL is altered as a consequence of enhanced triglyceride synthesis. Especially in marked hyperglycaemia, large and triglyceride-rich VLDL particles exist; but the conversion to LDL is diminished [68]. This may explain why LDL concentrations are normal or even reduced in Type 2 diabetic patients.

The alterations in lipid composition and the glycosylation of LDL are the main causes of decreased receptorial removal in Type 2 diabetes mellitus. An increase of the triglyceride content of LDL influences 
the binding to skin fibroblasts; Hiramatsu et al. showed that the decreased binding is inversely related to the triglyceride/protein ratio measured in LDL [29]. Glycosylation of some $40 \%$ of the lysine residues in apoprotein $\mathrm{B}$ completely blocks the receptor-mediated uptake of LDL [69]. These observations were demonstrated with skin fibroblasts as well as on cell membranes [34].

Pathological apoprotein compositions have been found in Type 2 diabetes mellitus by several investigators. Fielding et al. reported on an abnormal lipoprotein fraction with a calculated particle density within the low density lipoprotein range [27]. This fraction, enriched in apoprotein $\mathrm{E}$ and free cholesterol, may contribute to the diminished cholesterol net transport from cell membranes to the plasma of Type 2 diabetic patients. Attempts have been made to remove the apoprotein $\mathrm{E}$ content of the plasma of such patients, which is markedly elevated [27]. Under in vitro circumstances the removal of apoprotein $\mathrm{E}$ completely restored the marked depression of cholesterol transport in plasma. Increased apoprotein $E$ concentration and abnormal lipoproteins enriched in apoprotein $\mathrm{E}$ may account for dyslipoproteinaemia yielding the increased risk of atherosclerosis in Type 2 diabetes mellitus.

The HDL-cholesterol levels in Type 2 diabetic patients are rather lower than normal [53, 70]; a decrease of $\mathrm{HDL}_{2}$-cholesterol is mainly responsible for this. Theoretically, the enhanced activity of lipase may account for the hypercatabolism of HDL, which should lead to an accelerated cholesterol transport from peripheral tissues to the liver [53]. This process, which is considered "anti-atherogenic" rather than "atherogenic" is not compatible with the high prevalence of atherosclerotic diseases found in Type 2 diabetes mellitus. The low HDL-cholesterol may in part also be the expression of an increased proportion of triglycerides in HDL [59]. Thus, the protein to cholesterol ratio in HDL is elevated. Studies in different Type 2 diabetic populations indicate that apoprotein A-I content is depleted [66].

In summary, the most striking alteration of lipoproteins in Type 2 diabetic patients is the elevation of VLDL concentration as a result of insufficient insulin action in concert with hyperinsulinism. There is an enhanced production of apoprotein B; but the LDL is in most cases not elevated, indicating the direct removal of this apoprotein from the VLDL/IDL pool. The decreased level of HDL may be indicative of an atherogenic factor, but may also be the result of a demonstrated increase of hepatic lipase.

\section{Conclusions}

Considerable evidence has accumulated pointing towards a higher prevalence of early atherosclerosis in diabetic patients as compared to age, sex and body weight matched non-diabetic control subjects [71]. There seems to be no doubt that alterations in lipoprotein composition and metabolism account to an (as yet) unknown degree for this phenomenon. In that respect it should be remembered that Type 1 and Type 2 diabetic patients differ in their lipoprotein patterns quite substantially: in Type 1 patients under optimal metabolic control, the lipoproteins considered to be most atherogenic, VLDL, IDL and LDL, do not differ strikingly from that of normal subjects; and the anti-atherogenic fraction HDL is usually even increased [72]. Thus, it might rather be the lipoprotein flux than the absolute amount which matters under those circumstances. Another point to consider is the degree of oxidation of LDL-lipids, which is higher in those patients; this is partly responsible for observed microangiopathy. Since similar mechanisms are also currently being discussed as causes for macroangiopathy, derangements in the eicosanoid metabolism, membrane stiffness and lipid peroxydes, in concert with platelet reactivity and monocyte/macrophage activation, may be the leading causes for atherosclerosis in Type 1 diabetes. Whether or not the peripheral hyperinsulinism, which certainly persists for many hours per day in "well controlled" patients, contributes additionally to the pathogenesis of atherosclerosis is an as yet unsetteled question [73]. The situation might be different in Type 1 diabetic patients suffering from nephropathy. Under these circumstances, secondary dyslipoproteinaemias are abundant, similarly to patients with kidney diseases free of diabetes. The alterations under these circumstances lead to an increase of the lipoprotein fractions, which are known to be mostly atherogenic (apoprotein Bcontaining lipoproteins); and a decrease of antiatherogenic HDL.

The situation in Type 2 diabetes is quite different. Here, most atherogenic lipoproteins of intermediate density (beta-VLDL, IDL) are abundant, and antiatherogenic HDL are reduced in their concentration. An aggravating factor is the glycation of LDL. These abnormalities not only lead to an increased uptake of apoprotein B-containing lipoproteins by a scavenger pathway, which in turn yields macrophage activation and foam cell formation, but also, as a consequence of the reduced HDL, are accompanied by an impairment of the reverse cholesterol transport.

There is no doubt about the fact that poor metabolic control in both, Type 1 as well as Type 2 diabetic patients, is associated with the development of atherogenic lipoprotein patterns in plasma. Thus, any attempts made at control must be not only for optimal glycaemic control, but also for a nearly physiological $24 \mathrm{~h}$ insulin profile. Whether or not such treatment may one day reduce the atherosclerosis risk of all diabetic patients to match that of non-diabetic subjects remains to be established. 
Acknowledgement. Parts of the studies cited in this article were sup ported by the Austrian Research Fonds.

\section{References}

1. Garcia MJ, McNamara PM, Gordon T, Kannel WB (1974) Morbidity and mortality in diabetes in the Framingham population. Diabetes 23: 105-111

2. Ganda OP (1980) Pathogenesis of macrovascular disease in the human diabetic. Diabetes 29: 931-942

3. Sorge F, Schwartzkopff W, Neuhaus GA (1976) Insulin response to oral glucose in patients with previous myocardial infarction and in patients with peripheral vascular disease: Hyperinsulinemia and its relationship to hypertriglyceridemia and overweight. Diabetes 25: 586-594

4. Bierman EL, Brunzell JD (1978) Interrelation of atherosclerosis, abnormal lipid metabolism, and diabetes mellitus. In: Ktzen HM, Mahler RI (eds) Advences in modern nutrition, Vol.7. Wiley, New York, pp 187-210

5. Bierman EL, Amaral AP, Belknap BH (1966) Hyperlipidemia and diabetes mellitus. Diabetes 15: 675-679

6. Howard BV, Savage PJ, Bennion LJ, Bennett PH (1978) Lipoprotein composition in diabetes mellitus. Atherosclerosis 30 : 153-162

7. Epstein FH, Ostrander LD, Johnson BC, Payne MW, Haynes NS, Keller JB, Francis TJ (1965) Epidemiological studies of cardiovascular disease in a total community-Tecumseh, Michigan. Ann Int Med 62: 1170-1187

8. Keen H, Rose G, Pyke D (1965) Blood sugar and aterial disease. Lancet II: $505-508$

9. National Diabetes Group (1979) The classification and diagnosis of diabetes mellitus and other categories of glucose intolerance. Diabetes 28: 1039-1057

10. Vaccoro O, Rivellese A, Riccardi G, Capaldo B, Tutino L, Annuzzi G, Mancini M (1984) Impaired glucose tolerance and risk factors for atherosclerosis. Arteriosclerosis 4: 592-597

11. Gibbons GF (1986) Hyperlipidaemia of diabetes. Clin Sci 71: 477-486

12. Risser TR, Reaven GM, Reaven EP (1978) Intestinal contribution to secretion of very low density lipoproteins into plasma. Am J Physiol 234: E277-E281

13. Risser TR, Reaven GM, Reaven EP (1978) Intestinal very low density lipoprotein secretion in insulin deficient rates. Diabetes 27: 902-908

14. Steiner GS, Poapst M, Davidson JK (1975) Production of chylomicron-like lipoproteins from endogenous lipid by intestine and liver in diabetic dogs. Diabetes 24: 263-271

15. Popper DA, Shiau Y-F, Reed M (1985) Role of small intestine in pathogenesis of hyperlipidemia in diabetic rats. Am J Physiol 249: G161-G167

16. Taskinen M-R, Nikkilä EA, Kuusi T, Harno K (1982) Lipoprotein lipase activity and serum lipoproteins in untreated type 2 (insulin-independent) diabetes associated with obesity. Diabetologia 22: 46-50

17. Taskinen M-R, Nikkilä EA (1979) Lipoprotein lipase activity of adipose tissue and skeletal muscle in insulin-deficient human diabetes. Diabetologia 17: 351-356

18. Nikkilä EA (1984) Plasma lipid and lipoprotein abnormalities in diabetes. In: Jarrett RJ (ed) Diabetes and heart disease. Elsevier, Amsterdam, pp 133-167

19. Bagdade JD, Porte D, Bierman EL (1967) Diabetic lipemia. A form of acquired fat-induced lipaemia. N Engl J Med 276: $427-433$

20. Harno KE, Nikkilä EA, Kuusi T (1980) Plasma HDL-cholesterol and postheparin plasma hepatic endothel lipase activity: relationship to obesity and non-insulin-dependent diabetes. Diabetologia 19: 281

21. Howard BV (1987) Lipoprotein metabolism in diabetes mellitus. J Lipid Res 28: 613-628
22. Breckenridge WC, Little JA, Alaupovic P, Wang CS, Kuksis A, Kakis S, Lindgren F, Gardiner G (1982) Lipoprotein abnormalities associated with a familial deficiency of hepatic lipase. Atherosclerosis $45: 161$

23. Packard CH-J, Shepherd J (1985) The pathophysiology of cholesterol metabolism in man. Klin Wochenschr 63: 344-351

24. Reaven GM, Olefsky JM, Farquhar JW (1972) Does hyperglycaemia or hyperinsulinaemia characterise the patients with chemical diabetes? Lancet I: 1247-1249

25. Reaven GM (1987) Non-insulin-dependent diabetes mellitus, abnormal lipoprotein metabolism, and atherosclerosis. Metabolism 36: $1-8$

26. Patsch W, Gotto AM, Patsch JR (1986) Effects of insulin on lipoprotein secretion in rat hepatocyte cultures. J Biol Chem 261: 9603-9606

27. Fielding CJ, Reaven GM, Fielding PE (1982) Human noninsulindependent diabetes: identification of a defect in plasma cholesterol transport normalized in in vivo by insulin and in vitro by selective immunoadsorption of apolipoprotein E. Proc Natl Acad Sci USA 79: 6365-6369

28. Fielding CJ, Reaven GM, Liu G, Fielding PE (1984) Increased free cholesterol in plasma low and very low density lipoproteins in non-insulin-dependent diabetes mellitus: its role in the inhibition of cholesteryl ester transfer. Proc Natl Acad Sci USA 81: 2512-2516

29. Hiramatsu K, Bierman EL, Chait A (1985) Metabolism of low density lipoproteins from patients with diabetic hypertriglyceridemia by cultured human skin fibroblasts. Diabetes 34: 8-14

30. Brownlee M, Vlassara H, Cerami A (1985) Nonenzymatic glycosylation products on collagen covalently trap low density lipoprotein. Diabetes 34: 938-941

31. Witztum JL, Mahoney EM, Branks MJ, Fisher M, Elam R, Steinberg $D$ (1982) Nonenzymatic glucosylation of low-density lipoprotein alters its biologic activity. Diabetes 31: 283-291

32. Steinbrechner URSP, Witztum JL (1984) Glucosylation of lowdensity-lipoprotein to an extent comparable to that seen in diabetes slows their catabolism. Diabetes 33: 130-134

33. Schleicher E, Deufel T, Wieland OH (1981) Non-enzymatic glycosylation of human serum lipoproteins. FEBS Letters 129: 1-4

34. Triau JE, Arbetter J, Schaefer EJ (1986) Impaired hepatocyte binding, uptake and degradation of glucosylation low-density lipoproteins. Biochim Biophys Acta 877: 359-365

35. Lyons TJ, Baynes JW, Patrik JS, Colwell JA, Lopes-Virella MF (1986) Glycosylation of low density lipoprotein in patients with Type 1 (insulin-dependent) diabetes: correlations with other parameters of glycaemic control. Diabetologia 29: 685-689

36. Briones ER, Mao SJT, Palumbo PJ, O'Fallon WM, Chenoweth W, Kottke BA (1984) Analysis of plasma lipids and apolipoproteins in insulin-dependent and noninsulin-dependent diabetics. Metabolism 33: 42-49

37. Eto M, Watanabe K, Iwishima Y, Morikawa A, Oshima E, Sekiguchi M, Iski K (1986) Apolipoprotein E polymorphismus and hyperlipaemia in type II diabetics. Diabetes $35: 1374-1382$

38. Gonen B, White N, Schonfeld G, Skor D, Miller P, Santiago J (1985) Plasma levels of apoprotein B in patients with diabetes mellitus; the effect of glycemic control. Metabolism 34: 675-679

39. Weisweiler $P$, Jüngst $D$, Schwandt $P$ (1983) Quantitation of apolipoprotein E-isoforms in diabetes mellitus. Horm Metabol Res 15: 201

40. Maruhama Y, Saito F, Hikichi I, Hashimoto T, Takahashi K, Kaito I (1982) Apoprotein B-poor very low density lipoprotein in diabetic patients without hyperlipoproteinemia. Tohoku J Exp Med 138: 419-425

41. Stalenhoef AFH, Demacker PNM, Lutterman JA, van't Lear A (1982) Apolipoprotein C in type 2 (non-insulin-dependent) diabetic patients with hypertriglyceridemia. Diabetologia 22: 489-491

42. Fielding CJ, Castro GR, Donner C, Fielding PE, Reaven GM (1986) Distribution of apolipoprotein $E$ in the plasma of insulindependent and noninsulin-dependent diabetics and its relation to cholesterol net transport. J Lipid Res 27: 1052-1061 
43. Weisweiler P, Schwandt P (1987) Type 1 (insulin-dependent) versus type 2 (non-insulin-dependent) diabetes mellitus: characterization of serum lipoprotein alterations. Eur $\mathbf{J}$ Clin Invest 17: $87-91$

44. Nikkilä EA, Huttunen JK, Ehnholm C (1977) Postheparin plasma lipoprotein lipase and hepatic lipase in diabetes mellitus. Diabetes 26: 11-21

45. Nikkilä EA, Kekki M (1973) Plasma triglyceride transport kinetics in diabetes mellitus. Metabolism 22: 1-22

46. Owen OE, Block BSB, Patel M, Boden G, McDonough, Kreulen T, Shuman CR, Reichard GA (1977) Human splanchnic metabolism during diabetic ketoacidosis. Metabolism 26: 381-398

47. Lopes-Virella MF, Sherer GK, Lees AM, Wohltmann H, Mayfield R, Sagel J, LeRoy AC, Colwell JA (1982) Surface binding, internalization and degradation by cultured human fibroblasts of low density lipoproteins isolated from type I (insulin-dependent) diabetic patients: changes with metabolic control. Diabetologia 22: $430-436$

48. Pietri A, Dunn FL, Raskin P (1980) The effect of improved diabetic controll on plasma lipid and lipoprotein levels. Diabetes 29: $1001-1005$

49. Kennedy AL, Lappin TRJ, Lavery JD, Hadden Dr, Weaver JA, Montgomery DAD (1978) Relation of high density lipoprotein cholesterol concentration to type of diabetes and its control. $\mathrm{Br}$ Med J 2: 1191-1194

50. Nikkilä EA, Hormila P (1978) Serum lipids and lipoproteins in insulin-treated diabetics. Demonstration of increased high density lipoprotein concentrations. Diabetes 27: 1078-1085

51. Laakso M, Pyörälä K, Sarlund H, Voutilainen E (1986) Lipid and lipoprotein abnormalities associated with coronary heart disease in patients with insulin-dependent diabetes mellitus. Arteriosclerosis 6: 679-684

52. Mattock MB, Fuller JH, Maude PS, Keen H (1979) Lipoproteins and plasma cholesterol esterification in normal and diabetic subjects. Atherosclerosis 34: 437-449

53. Nikkilä EA (1985) Are plasma lipoproteins responsible for the excess atherosclerosis in diabetes? Acta Endocrinol [Suppl] 110: 272: $27-30$

54. Nikkilä EA (1981) High density lipoproteins in diabetes. Diabetes $30: 82-87$

55. Durrington PN (1982) Serum high density lipoprotein cholesterol subfractions in type I (insulin-dependent) diabetes mellitus. Clin Chim Acta 120: 21-28

56. Taskinen MR, Kuusi T, Nikkilä EA (1985) Regulation of HDL and its subfractions in chronically insulintreated patients with type I diabetes. In: Crepaldi G, Tiengo A, Baggio G (eds) Diabetes, Obesity and Hyperlipidaemias. Elsevier, Amsterdam, pp251-259

57. Kobbah M, Vessby B, Tuvemo T (1988) Serum lipids and apolipoproteins in children with insulin-dependent diabetes during the first two years of the disease. Diabetologia 31: 195-200

58. Tall AR, Small DM (1978) Plasma high density lipoproteins. N Engl J Med 299: 1232-1236

59. Chang LB, Hopkins FGJ, Bartel PJ (1985) Particle size distribution on high density lipoproteins as a function of plasma trigly- ceride concentration in human subjects. Atherosclerosis 56: 61-70

60. Scherthaner G, Kostner GM, Dieplinger H, Prager H, Muhlhauser I (1983) Apolipoproteins (A-I, A-II, B), Lp/a/ lipoprotein and lecithin: cholesterol acyltransferase activity in diabetes mellitus. Atherosclerosis 49: 277-293

61. Jensen T, Stender S, Deckert T (1988) Abnormalities in plasmas concentrations of lipoproteins and fibrinogen in Type 1 (insulindependent) diabetic patients with increased urinary albumin secretion. Diabetologia 31: 142-145

62. Brunzell JD, Porte DD, Bierman EL (1975) Reversible abnormalities in postheparin lipolytic activity during the late phase of release in diabetes mellitus (postheparin lipolytic activity in diabetes). Metabolism 24: 1123-1137

63. Vessby B, Selinus I, Lithell H (1985) Serum lipoprotein and lipoprotein lipase in overweight, type II diabetics during and after supplemented fasting. Arteriosclerosis 5:93-100

64. Howard BV, Davis M, Pettitt DJ, Knowler WC, Bennett PH (1983) Plasma and lipoprotein cholesterol and triglyceride concentrations in the Pima Indians: distribution differing from those of Caucasians. Circulation 68: 214-222

65. Chait A, Robertson HT, Brunzell JD (1981) Chylomicronaemia syndrome in diabetes mellitus. Diabetes Care 4: 343-348

66. Gabor J, Spain M, Kalant N (1980) Composition of serum verylow-density and high-density lipoproteins in diabetics. Clin Chem 26: 1261-1265

67. Quarfordt SH, Michalopoulos G, Schirmer B (1982) The effect of human $\mathrm{C}$ apolipoproteins on the in vitro hepatic metabolism of triglyceride emulsions in the rat. J Biol Chem 257: 14642-14647

68. Kissebah AH, Alfarsi S, Evans DJ, Adams PW (1982) Integrated regulation of very low density lipoproteins in hyperlipidaemia: studies of apolipoprotein-B kinetics in non-insulin-dependent diabetes mellitus. Diabetes 31:217-225

69. Kesaniemi YA, Witztum JL, Steinbrecher UP (1983) Receptormediated catabolism of low density lipoprotein in man. Quantitation using glucosylated low density lipoprotein. J Clin Invest 71: 950-959

70. Barrett-Connor E, Witztum J, Hodbrook M (1983) A community study of high density lipoproteins in adult noninsulin-dependent diabetics. Am J Epidemiol 117: 186-192

71. Schonfeld G (1985) Diabetes, lipoproteins, and atherosclerosis. Metabolism 34: 45-50

72. Romics L, Karádi I, Rozenberczki M, Kammerer L (1982) Effect of metabolic control of the serum lipid and lipoprotein levels on insulin-dependent diabetes mellitus. Acta med. Acad Sci Hung 39: $117-123$

73. Jarret RJ (1988) Is insulin atherogenic? Diabetologia 31: 71-75

Received: 25 July 1988

Prof. G. M. Kostner

Institute of Medical Biochemistry

University of Graz

Harrachgasse 21/III

A-8010 Graz

Austria 